\title{
¿tiene incidencia la pobreza infantil y juvenil en los resultados académicos?*
}

\author{
Joan Tahull Fort* \\ Fidel Molina Luque ${ }^{* * *}$ \\ Iolanda Montero Plaza****
}

Recibido: 17 de enero de 2016 - Aprobado: 25 de mayo de 2016

Este estudio presenta una descripción, análisis y reflexión sobre la pobreza infantil y juvenil. España enfrenta una crisis económica muy profunda, y las propuestas del Gobierno para abordar los problemas sociales consisten fundamentalmente en avanzar en el modelo neoliberal. Los alumnos van a la escuela, pero en muchos casos sus resultados son deficientes, lo que hace que España presente un alto índice de fracaso escolar. La metodología incluye investigación bibliográfica, tratamiento de datos cuantitativos y cualitativos y entrevistas en profundidad. Los resultados obtenidos muestran un abandono escolar muy alto, junto con un número significativo de niños y adolescentes en situación de pobreza, lo cual permite establecer una relación entre pobreza infantil y juvenil y abandono escolar. El Gobierno centra su atención en la institución educativa y solo consigue desacreditarla; sus actuaciones y resultados son insuficientes. Por ello, la propuesta es que - junto con las autoridades e instituciones pertinentes - implemente políticas globales e integrales para abordar el problema.

Palabras clave: familia, exclusión social, crisis económica, educación y sociedad.

Artículo de investigación científica y de desarrollo tecnológico, producto de los intereses y motivaciones del Grupo de Estudios sobre Sociedad, Salud, Educación y Cultura (Gesec), Grupo de Investigación Consolidado y financiado por la Agaur (2014SGR655), y adscrito a la Universidad de Lleida (España). No ha recibido ningún tipo de financiación. DOI: http://dx.doi.org/10.15332/s0120-8454.2017.0090.02

* Doctor en Sociología por la Universidad de Lleida, licenciado en Filosofía y en Antropología Social y Cultural. Profesor del Departamento de Sociología y Geografía de la Universidad de Lleida. Profesor de secundaria, Generalitat de Catalunya. Miembro del Grupo de Estudios sobre Sociedad, Salud, Educación y Cultura (Gesec). Dirección postal: calle Divina Pastora 1 Balaguer (Lleida) 25600. Correo electrónico: joantfort@geosoc.udl.cat

**- Catedrático de Sociología de la Universidad de Lleida. Miembro del Grupo de Estudios sobre Sociedad, Salud, Educación y Cultura (Gesec). Director del Instituto de Investigación de Desarrollo Social y Territorial (Indest). Dirección postal: avenida Estudi General 4 Lleida 25001 (España). Correo electrónico: molina@geosoc.udl.cat

*tr* Antropóloga y psicopedagoga. Profesora de secundaria, Generalitat de Catalunya. Dirección postal: calle Divina Pastora 1 Balaguer (Lleida) 25600. Correo electrónico: ymontero@xtec.cat 


\title{
Family malaise: does child and youth poverty impact academic performance?*
}

\author{
Joan Tahull Fort ${ }^{* *}$ \\ Fidel Molina Luque ${ }^{* * *}$ \\ Iolanda Montero Plaza***
}

Abstract

This research is a description, analysis and reflection on child and youth poverty. Spain has a very deep economic crisis. Proposals for the Government to address social problems are based on the neoliberal model. Students go to school, but in many cases the results are poor, so Spain presents a high rate of school failure. The methodology includes bibliographic research, interviews in depth and treatment of quantitative and qualitative data. The results show a very high dropout. In Spain, there is a significant number of children and adolescents in situation of poverty, which allows to establish a relationship between child and youth poverty and dropout. The Government focuses its attention on the educational institution and only gets to discredit it; performances and results are insufficient. Therefore, the proposal is that the Ministry of education (together with other relevant institutions) develop global and comprehensive policies in order to deal with the problem.

Keywords: Family, social exclusion, economic crisis, education and society

Scientific research and technological development article, resulting from the interests and motivations of the Study Group on Society, Health, Education and Culture ( $\mathrm{Gesec}$ ), Consolidated Research Group and financed by the Agaur (2014SGR655), and attached to the University of Lleida (Spain). Has not received any funding. DOI: http://dx.doi. org/10.15332/s0120-8454.2017.0090.02

* $\quad \mathrm{PhD}$ in Sociology from the University of Lleida, graduated in Philosophy and Social and Cultural Anthropology. Professor of the Department of Sociology and Geography of the University of Lleida. High school teacher, Generalitat de Catalunya. Member of the Study Group on Society, Health, Education and Culture (Gesec). Postal address: Calle Divina Pastora 1 Balaguer (Lleida) 25600. Email: joantfort@geosoc.udl.cat

** Professor of Sociology at the University of Lleida. Member of the Study Group on Society, Health, Education and Culture (Gesec). Director of the Research Institute for Social and Territorial Development (Indest). Postal address: avenida Estudi General 4 Lleida 25001 (Spain). Email: molina@geosoc.udl.cat

Anthropologist and psychopedagogue. High school teacher, Generalitat de Catalunya. Postal address: calle Divina Pastora 1 Balaguer (Lleida) 25600. Email: ymontero@xtec.cat 


\title{
Malaise familial: la pauvreté infantile et juvénile a-t-elle une incidence sur les résultats académiques ${ }^{*}$
}

\author{
Joan Tahull Fort ${ }^{* *}$ \\ Fidel Molina Luque*** \\ Iolanda Montero Plaza****
}

\section{Résumé}

Cette étude présente une description, une analyse et une réflexion sur la pauvreté infantile et juvénile. L'Espagne fait face à une grave crise économique, et les propositions du Gouvernement pour aborder les problèmes sociaux consistent fondamentalement à avancer dans le modèle néolibéral. Les élèves vont à l'école, mais dans beaucoup de cas leurs résultats sont mauvais, ce qui fait qu'il existe un taux élevé d'échec scolaire en Espagne. La méthodologie inclut une recherche bibliographique, un traitement de données quantitatives et qualitatives et des interviews en profondeur. Les résultats obtenus montrent un décrochage scolaire très élevé, avec un nombre significatif d'enfants et d'adolescents en situation de pauvreté, ce qui permet d'établir une relation entre pauvreté infantile et juvénile et désertion scolaire. Le Gouvernement met l'accent sur l'institution éducative et ne réussit qu'à la discréditer; ses rôles et ses résultats sont insuffisants. C'est pour cela que la proposition est que - avec les autorités et les institutions pertinentes - il mette en application des politiques globales et intégrales pour aborder le problème.

Mots clés: Famille, exclusion sociale, crise économique, éducation et société

Article de recherche scientifique et de développement technologique, résultat des intérêts et motivations du Groupe d’Études sur la Société, Santé, Éducation et Culture (Gesec), Groupe de Recherche Consolidé et financé par l'Agaur (2014SGR655), et adscrit à I'Université de Lleida (Espagne). Elle n'a reçu aucun type de subvention. DOI: http://dx.doi. org/10.15332/s0120-8454.2017.0090.02

* Docteur en Sociologie de I'Université de Lleida, licencié en Philosophie y Anthropologie Sociale et Culturelle. Professeur du Département de Sociologie y Géographie de l'Université de Lleida. Professeur de collège, Generalitat de Catalunya. Membre du Groupe d’Études sur la Société, Santé, Éducation et Culture (Gesec). Adresse postale: calle Divina Pastora 1 Balaguer (Lleida) 25600. Email: joantfort@geosoc.udl.cat

*** Professeur de Sociologie de l'Université de Lleida. Membre du Groupe d'Études sur la Société, Santé, Éducation et Culture (Gesec). Directeur de I'Institut de Recherche de Développement Social et Territorial (Indest). Adresse postale: avenida Estudi General 4 Lleida 25001 (Espagne). Email: molina@geosoc.udl.cat

Anthropologue et psychopédagogue. Professeur de collège, Generalitat de Catalunya. Adresse postale: calle Divina Pastora 1 Balaguer (Lleida) 25600. Email: ymontero@xtec.cat 


\section{Introducción}

Este artículo presenta una reflexión sobre la pobreza infantil y juvenil en España, particularmente en Cataluña, en un contexto social, cultural y económico neoliberal. Se muestra una visión global de la pobreza, desde diferentes puntos de vista, y se analiza su relación con la educación.

Los diferentes países de la Unión Europea, incluida España, enfrentan una larga y profunda crisis económica. El desempleo es uno de los principales problemas, y no se vislumbran cambios significativos en un futuro próximo. Esta situación tiene muchas consecuencias negativas y es inaceptable a escala social, por lo cual debería abordarse de forma rigurosa. Las condiciones sociales y económicas no mejoran, más bien empeoran, y las familias, sobre todo los más vulnerables, los hijos, sufren esta situación de desamparo. Es necesario un abordaje global e integral del desempleo, a cargo de todos los organismos e instituciones relacionadas, pero esto no sucede. Por el contrario, se aplican políticas económicas neoliberales de contención del gasto público y de privatización de servicios básicos, a la vez que se sigue un proceso lento e inexorable de reducción de políticas de protección social.

Los niños y jóvenes hijos de familias vulnerables que están en edad de escolarización obligatoria enfrentan situaciones - usualmente complejas y desagradables - que repercuten en su rendimiento académico. En España, el fracaso escolar está muy por encima de la media de los países de la Organización para la Cooperación y el Desarrollo Económicos (OCDE), y el país se sitúa a la cabeza de la Unión Europea cuando se trata de abandono escolar ${ }^{1}$. Amplios sectores sociales, élites, intelectuales, periodistas, tertulianos... consideran que la educación necesita un cambio radical. Se le acusa de no estar adaptada a los tiempos, de tener una organización del siglo XIX en una sociedad del siglo XXI. Pero sin profundizar en estas afirmaciones, que en algunos aspectos pueden tenerse en cuenta, lo que se muestra aquí es una realidad social y cultural problemática incrustada en los centros educativos: la pobreza infantil y juvenil.

El artículo busca ofrecer una visión panorámica de la realidad social y cultural de muchas familias españolas y catalanas. Una sociedad avanzada, del conocimiento, exige una educación moderna y adaptada a los tiempos. Muchas veces la educación es solo aquello que sucede en el interior de los centros educativos, pero los conflictos sociales que están más allá de las escuelas influyen significativamente en el proceso de aprendizaje de los niños.

Élites, políticos e intelectuales reiteran que la educación no cumple, que tiene muchas carencias y que necesita un cambio radical de modelo. Todos ellos quieren "poner patas arriba" la estructura educativa, pero sus análisis no tienen en cuenta su relación con el entorno; hablan de la educación como si fuese una

1 Véase la noticia en el diario El País, del 11 de abril de 2014, en el siguiente enlace: http://sociedad.elpais.com/sociedad/2014/04/11/actualidad/1397211917_985641.html 
mónada, un ente aislado y cerrado en sí mismo. Muchos aportes carecen de una perspectiva social y cultural y, sobre todo, de la situación de pobreza de muchas familias con hijos.

Se afirma que la educación es la mejor forma de solucionar la profunda crisis social y económica que enfrenta el país, pero no se tienen en cuenta sus limitaciones. Hay desajustes sociales que van más allá de los centros educativos, por lo cual se requiere la implementación de políticas más amplias y profundas. La pobreza infantil y juvenil no solo es un problema en el ámbito educativo, sino también en las esferas social y económica, y requiere, por lo tanto, políticas integrales para su abordaje. Como bien lo dice el proverbio africano, "Para educar a un niño hace falta la tribu entera". La escuela no debe encargarse sola de la educación de los niños.

Muchos adultos, también jóvenes, no reciben una capacitación adecuada para adaptarse a los cambios sociales, y no cuentan con las herramientas necesarias para enfrentar las dificultades y los retos de la sociedad española. El fracaso escolar es muy elevado en España, así como las tasas de pobreza infantil y juvenil. Si bien la educación es un ámbito muy estudiado, desde diferentes materias y puntos de vista, dado el contexto señalado, la relación entre pobreza y éxito o fracaso escolar requiere atención. La sociología y el trabajo social abordan la exclusión y diferentes formas de discriminación social, pero resulta difícil encontrar investigaciones adecuadas que expresen la relación entre pobreza y educación.

\section{Fundamentos teóricos}

Vivimos en un contexto neoliberal, caracterizado por la globalización, y los ciudadanos deben afrontar retos inmensos para adaptarse a la sociedad, pues la incertidumbre y el riesgo están incrustados en la estructura social y cultural. Estos rasgos genéricos no son propios de un país o lugar específico, sino de todos, en mayor o menor intensidad. Los sociólogos caracterizan la posmodernidad mediante diferentes conceptos significativos, pero siempre bajo un común denominador: las dificultades, el caos, la fragmentación, la complejidad y el desorden.

Bauman (2003) señala que la realidad social se presenta de forma líquida: las estructuras, interacciones sociales y conceptos son inestables; el sujeto no tiene elementos de referencia fijos para orientarse. Giddens (2000) destaca el papel del Estado. En el pasado brindaba seguridad a los ciudadanos, pero en la actualidad ha perdido parte de su soberanía, delegada a corporaciones privadas. El estado de bienestar pierde relevancia. El individuo está desorientado y vive en un estado liminal. Lipovetsky (2008) afirma que vivimos en la sociedad de la decepción; no hay relatos significativos para una mayoría de ciudadanos; el sujeto está regido por el vacío; lo importante es la forma y la estética, no el fondo. Según Beck (1998), asistimos a la irrupción de lo precario, discontinuo, 
impreciso e informal. La inseguridad es un elemento fundamental para comprender el presente. Elzo (2000) resalta los cambios en la organización familiar y la aparición de múltiples tipologías. Muchas familias tienen dificultades económicas y todos sus miembros sufren las consecuencias. McLuhan (2005) destaca el concepto de globalización, a partir de un mundo que se ha hecho pequeño, e introduce la metáfora de la aldea global. El mundo es parecido a una aldea; grandes flujos de personas se trasladan a otros lugares para buscar una vida mejor. El autor también destaca el individualismo o la relevancia del individuo frente a la comunidad. Al respecto, Giddens (2000) habla de una pérdida del sentido colectivo. El sujeto defiende sus intereses por encima de las reivindicaciones comunitarias. Los individuos están más aislados y separados, y esto ocurre en diferentes ámbitos: social, empresarial y familiar.

La situación social y cultural actual se caracteriza por la incertidumbre. En España hay una larga y profunda crisis económica (una consecuencia importante es el paro ${ }^{2}$ ); muchas familias están en una situación límite, en la pobreza, en la exclusión social, lo cual tiene consecuencias en diferentes ámbitos: familiar, económico, social, psicológico.

Si bien las élites de la Unión Europea, incluidas las de España, han impuesto diferentes políticas de contención y reducción del gasto público, los políticos, los intelectuales y los comentaristas consideran que reformar la educación es la única alternativa para mejorar la situación. Por esta razón, un debate vivo e intenso se ha desplegado en torno a este problema, desde diferentes perspectivas. Un aspecto destacado es el fracaso escolar ${ }^{3}$, principalmente por su elevada tasa en España. Según datos de Fernández Enguita (2010), en el periodo 2006-2007, el porcentaje de alumnos egresados de la educación obligatoria sin título fue del $28,4 \%$ en España y del 23,6 \% en Cataluña. Según un informe publicado por la oficina estadística de la Unión Europea, Eurostat, en 2014, la tasa de abandono prematuro en España de la población total de 18 a 24 años - que completaron, como mucho, el primer ciclo de secundaria - fue del 21,9\%. Este porcentaje duplica la media comunitaria, la cual está en $11,1 \%$.

España es el país de la Unión Europea con el índice de abandono prematuro más elevado. Fernández Enguita alerta sobre los riesgos de esta situación: quienes obtengan el máximo de su formación inicial accederán, por ello, a los empleos más enriquecedores (en términos de cualificación y de desarrollo personal, además del salario, las condiciones de trabajo y otras contrapartidas) y tendrán más y mejores oportunidades de formación ulterior (capacitaciones laborales o estudios de posgrado con o sin apoyo de su empleador). En cambio, quienes

2 La tasa de desempleo en España en 2013 fue del 25,73 \%; en 2014, del 23,70\%, y en 2015, del 22,3 \%, según la Encuesta de Población Activa (EPA) que confecciona el Instituto Nacional de Estadística (INE), organismo autónomo de España encargado de la coordinación general de los servicios estadísticos de la Administración General del Estado.

3 Existen diferentes definiciones de fracaso escolar. Algunos autores consideran que es la no culminación de la educación obligatoria (ESO); otros, la no finalización de la educación postobligatoria. El fracaso escolar hace referencia a la no integración laboral en la sociedad. Fernández Enguita (2010), entre otros, opta por una concepción amplia del término, e incluye la no consecución de los objetivos mínimos razonables proclamados por la sociedad para integrarse en el mercado laboral, lo que en España equivale al título de educación obligatoria (ESO). 
desaprovechen esa formación inicial o no logren beneficiarse de ella tendrán más probabilidades de acabar en el desempleo o en puestos de trabajo poco cualificados (2010, p. 17).

La situación del alumno fracasado, aquel sin las credenciales educativas mínimas necesarias, es poco halagüeña. El hecho de no poseer el título básico y obligatorio le impide acceder a una formación posterior, posobligatoria, por lo cual se estanca y no puede progresar. Esta situación produce una brecha enorme (casi imposible de equilibrar en el futuro) entre jóvenes como este y aquellos que continúan avanzando hacia credenciales educativas superiores. En una sociedad del conocimiento, la educación no es un lujo, sino una necesidad básica de todos los individuos, ya que les permite ser, comprender, trabajar, interpretar, relacionarse... La formación permite la integración del sujeto en la estructura social y cultural. Entonces, ¿qué sucede con los fracasados escolares?, ¿con aquellos que no tienen los mínimos conocimientos para integrarse en todos los niveles en una sociedad compleja?

Los retos de los alumnos, futuros adultos, en una sociedad postmoderna son enormes. Deben tener capacidad de adaptación en entornos fragmentados, complejos, dinámicos y diversos. La escuela tiene la función de enseñar, educar y capacitar a todos los alumnos. Recibe críticas por no estar a la altura de las circunstancias, por ser una institución del siglo XIX, en medio de una sociedad del siglo XXI. Se la culpa del fracaso escolar, pero también de todos los males sociales: todo aquello negativo de la sociedad se origina en la escuela, en la mala educación recibida y en la decadencia de la institución, por lo cual se propone una reinvención de la educación.

La relación entre pobreza y educación ha sido escasamente abordada. Hay múltiples estudios sobre la escuela y la pobreza por separado, pero resulta difícil encontrar investigaciones rigurosas actuales que las relacionen. Uno de los objetivos de este artículo consiste en relacionar estos ámbitos, en comprender la educación desde el trabajo social, conectar estas disciplinas y abrir el campo de reflexión a una mirada diferente.

La escuela tiene la función de formar y educar a todos los niños en unos contenidos aceptados por la sociedad, pero las capacidades de cada familia para acompañar a sus hijos son muy desiguales; las diferencias sociales, culturales, religiosas, étnicas, económicas ponen en entredicho aquella idea de igualdad de oportunidades, que en muchos casos resulta ser una ilusión, una quimera. En este contexto de pobreza ${ }^{4}$ y exclusión social ${ }^{5}$, los niños son el colectivo más perjudicado. Según Allepuz (2014, p. 172) en España, “En los últimos años ha

4 El término pobreza hace referencia a desigualdades económicas existentes entre personas y colectivos que viven en un mismo contexto social y económico. En situaciones de pobreza, una familia o individuo no recibe una determinada cantidad de dinero y está por debajo del mínimo considerado adecuado para vivir con dignidad.

5. En los últimos años ha aparecido el concepto de exclusión social en algunas disciplinas sociales. El término hace referencia a procesos de vulnerabilidad, rotura social, pérdida de vínculos sociales y familiares, relacionados con diversas variables de desigualdad y pobreza. Se presenta como el resultado de una acumulación y combinación de diferentes factores de desigualdad que afectan el desarrollo personal, social, cultural y político de las personas en su entorno social. 
disminuido el riesgo de pobreza de los jubilados y ha aumentado significativamente para los menores de edad [...]. La pobreza infantil es uno de los datos más preocupantes de la situación social actual y de futuro".

La pobreza y la exclusión social no son realidades sociales ajenas a los centros educativos; al contrario, los menores de edad son el colectivo más vulnerable. La escuela no es una mónada, aislada y alejada del contexto sociocultural y económico; en la escuela se incrustan los problemas sociales, y los niños los sufren en silencio, pues no tienen la capacidad de protestar y reivindicar sus derechos.

Maslow (1991), hace más de cincuenta años (aunque su pensamiento todavía es muy actual), desarrolló una teoría de la motivación humana y estableció una jerarquía de las necesidades, que considera que las personas tienen comportamientos diferentes en función del estado de satisfacción de sus necesidades. Así, cuando la necesidad básica y fundamental de todos los individuos (que es la alimentación) está satisfecha, se tienen otras motivaciones.

Puesto que las necesidades fisiológicas son las más potentes de todas las necesidades, es muy probable que estas se conviertan en la mayor motivación de un ser humano que carece de todo en la vida y que se ve en una situación extrema. Una persona que carece de alimento, seguridad, amor y estima, probablemente, sentirá con más fuerza el hambre de comida antes que de cualquier otra cosa (1991, p. 23).

El autor estadounidense comprende las conductas de los individuos en función de sus necesidades, y sitúa en la satisfacción de las necesidades fisiológicas la base del desarrollo intelectual. Lo más importante es la comida; por lo tanto, cuando hay necesidad alimentaria, todas las energías vitales buscan saciarla, y se desplazan otros aspectos. Según Maslow:

Si todas las necesidades están sin satisfacer, y el organismo, por tanto, está dominado por las necesidades fisiológicas, las restantes necesidades simplemente pueden ser inexistentes o ser desplazadas al fondo. [...] Para el ser humano que tiene hambre no existen otros intereses que no sea la comida. Esta persona sueña con la comida, recuerda la comida, piensa en la comida, se emociona sólo con la comida, percibe la comida y solo quiere comida. (p. 23)

Este planteamiento permite comprender algunos problemas de la educación en España. La crisis económica, con sus altas tasas de desempleo, tiene a muchas familias ${ }^{6}$ en estado de vulnerabilidad, debilidad e inseguridad, pero el Gobierno rehúye su responsabilidad y traslada a las familias y centros educativos todos los problemas sociales externos a la institución. Si bien las escuelas tienen la función

6 En algunas familias, sus miembros están desempleados y no reciben remuneración alguna; en otras, solo un adulto trabaja, y a cambio de un sueldo bajo; también hay familias monoparentales y otras tipologías familiares complejas. Existen demasiados casos de trabajadores con sueldos que no les permiten salir de la pobreza. 
social de construir alumnos responsables, creativos, tolerantes e inteligentes, el abordaje de la política educativa es un acto complejo y dinámico, en el que intervienen múltiples ámbitos (social, cultural, económico y educativo); estas aportaciones y cambios no deben implementarse desde una única institución, la escuela, sino desde todos los organismos implicados, en la forma de políticas integrales coordinadas. Hargreaves (1999) considera que la escuela está en una situación muy complicada, pues se le otorga la responsabilidad de solucionar cuestiones sociales y culturales que están más allá de sus posibilidades. En muchos casos, el fracaso está asegurado y provoca un descrédito mayor de la institución:

En momentos de crisis, la educación en general y las escuelas en particular se convierten en lo que A. H. Haysey llamó una vez "la papelera de la sociedad": receptáculos políticos en los que se deposita sin ceremonia alguna lo no resuelto de la sociedad y los problemas insolubles. Pocas personas desean hacer algo respecto a la economía, pero todo el mundo (políticos, periodistas...) quieren hacer algo en educación (p. 31).

El autor cuestiona el verdadero interés de las élites para mejorar la educación. Si bien abordar mejoras en algunos ámbitos es problemático y tiene costes económicos y políticos, actuar en la escuela es muy fácil y resulta poco costoso política y monetariamente. Se introducen cambios en la educación, con poca o ninguna significación social y cultural.

En España, y en los países occidentales en general, la educación enfrenta un momento de crisis: se la considera responsable de los males de la sociedad y se culpa a los profesionales de no hacer su trabajo; además, se le otorga el deber de abordar y solucionar problemas complejos, pero no cuenta con los instrumentos adecuados para ello y, en consecuencia, sigue un proceso de continuo descrédito. Pierde reconocimiento, autoridad y prestigio social; se convierte, tal como lo manifiestan Halsey, Heath y Ridge (1980), en "la papelera de la sociedad" (p. 227). Todo aquello de difícil solución es tarea escolar, sin tener el apoyo suficiente del Estado, las élites y la sociedad. Según Terrén (1999, p. 227),

Hoy día no hay $[. .$.$] candidato electoral que no haga de la reforma de la$ educación uno de los puntos fuertes de su programa de gobierno. [...] A veces incluso podría pensarse que el paisaje de crisis es un cuadro de sentimientos inducidos (en gran parte mediáticamente) para justificar medidas cuyo rendimiento político poco tiene que ver con la educación.

El autor español sitúa la educación como una prioridad para los políticos, aunque su interés principal es propagandístico. En muchas ocasiones, sus objetivos no son mejorar la sociedad o la educación, sino obtener réditos personales. Las élites consideran los conflictos sociales deficiencias educativas (la escuela no realiza su trabajo de manera correcta). Se insiste en su importancia, pero sin dotarla de los instrumentos necesarios para que pueda realizar su trabajo. 
La pobreza de las familias (también de sus hijos) es relevante en el presente y para las generaciones futuras. Como afirma Giddens (2000), el estado de bienestar está en retroceso y limita progresivamente la confianza y las prestaciones de los ciudadanos. Por este motivo, sin un cambio radical de modelo económico, será muy difícil invertir la situación. Los niños son los más perjudicados y vulnerables. Marí-Klose y Marí-Klose (2012) explican algunas consecuencias de la pobreza en las familias:

La vulnerabilidad económica no solamente tiene una dimensión monetaria. Algunos de los efectos más importantes provocados por la exclusión económica tienen que ver con estados de malestar e inseguridad que están en la escasez de los recursos económicos y la incapacidad de generarlos. Los hijos no [son los únicos que] sufren las limitaciones económicas; también, y no menos importante, [estas] afectan la calidad de las relaciones entre los adultos de casa y estos con sus hijos. Los padres que sufren dificultades financieras están más irascibles y estresados y tienden a reaccionar de forma más inconsciente e incontrolada. (p. 11)

Esas consecuencias de la pobreza, según las autoras, van más allá de la escasez material. Los problemas económicos tienen consecuencias psicológicas; generan malestar e inseguridad en los adultos, y este estado se traslada a sus hijos. La relación con ellos se resiente, tiende a ser peor, más tensa y conflictiva. Los adultos pierden la paciencia más fácilmente. Marí-Klose (2012) y Marí-Klose citan a Griggs y Walker (2008) y afirman que "la pobreza infantil influye en el desarrollo cognitivo y los resultados educativos y facilita comportamientos asociales" (p. 4).

\section{Metodología}

Para la realización de este artículo se ha utilizado información cuantitativa del Instituto Nacional de Estadística (INE), recogida en la Encuesta de Condiciones de Vida (ECV). Se ha compilado datos significativos en las diferentes tablas sobre riesgo de pobreza o exclusión social por comunidades autónomas, tasa de riesgo de pobreza por tipo de hogares, brecha de riesgo de pobreza, abandono educativo temprano, tasa de abandono según quintil de renta disponible per cápita del hogar en 2010 y tasa de abandono según la capacidad del hogar de llegar a final de mes. Todas tienen un denominador común que permite conocer numéricamente aspectos relevantes del estado global de las familias españolas y la relación de los niños con la educación.

Los datos cualitativos ${ }^{7}$ complementaron la información cuantitativa, porque las metodologías utilizadas para tal fin (grupos de discusión, grupos triangu-

La información cualitativa se ha extraído de la tesis doctoral de Tahull (2012). 
lares, entrevistas en profundidad, observación y consulta de diferentes fuentes documentales) permiten describir y profundizar en ámbitos más concretos y específicos. Se buscaron informantes que pudieran ofrecer una mirada concreta y profunda del objeto de estudio. Se realizó la investigación cualitativa de una comunidad autónoma española, Cataluña ${ }^{8}$, a partir de las reflexiones de profesores, padres y alumnos de las cuatro provincias catalanas. La distribución de los participantes se realizó con base en diferentes criterios: rural/urbano, hombre/mujer, escuela pública/concertada, diferentes edades de los profesores, padres y alumnos. Todos los informantes son anónimos: personas que si bien tienen una experiencia relevante para este estudio, no aparecen en los medios de comunicación ni tienen una relevancia pública destacada.

Un problema importante en todas las investigaciones, también en esta, es la subjetividad. Se interpreta y describe la realidad social a partir del color de un lente particular. Como docentes, el contacto continuo con niños y jóvenes les ha permitido a los autores de este artículo leer, hablar, discutir y reflexionar sobre ellos, para así construir un relato más sólido.

La subjetividad no debe verse como una limitación o una parcialidad, al contrario, es un elemento propio e interesante del objeto de estudio; ofrece miradas y reflexiones que de otra manera no verían la luz. En todo caso, no todos los contenidos tienen el mismo valor. El investigador debe triangular la información, es decir, ponderar, valorar, situar, contrastar y comparar todos los datos (Stake, 1998; Ander Egg, 2000).

Para la investigación se establecieron dos grupos triangulares y un grupo de discusión. El primer grupo está integrado por un alumno de primero de bachillerato, una alumna de segundo de bachillerato y una alumna del primer ciclo de grado superior de formación profesional de Educación Infantil. Los tres pertenecen al mismo instituto, en la ciudad de Lleida. El segundo grupo son tres profesores de Barcelona y zonas próximas; todos trabajan en centros públicos y tienen una larga experiencia. El grupo de discusión está integrado por cinco profesores de un instituto en Tarragona y por el equipo directivo del centro: la directora, el jefe de estudios, la secretaria, la coordinadora pedagógica y un profesor. Todos son funcionarios (entre 45 y 55 años de edad), con una larga experiencia en diferentes centros públicos.

La siguiente técnica de investigación utilizada fue la entrevista en profundidad. Se buscaron las mejores condiciones para que los entrevistados (cuatro profesores de las cuatro provincias catalanas, dos alumnos del instituto de Lleida y Tarragona, respectivamente, y dos madres de Lleida y Girona) pudieran expresar con libertad y confianza sus opiniones. Entre los diferentes perfiles, se

8 Cataluña es una comunidad autónoma española, considerada nacionalidad histórica. Situada al noreste de la península Ibérica, está formada por cuatro provincias: Barcelona, Girona, Lleida y Tarragona. Su capital es la ciudad de Barcelona. Limita al norte con Francia y Andorra, al este con el mar Mediterráneo, al sur con la comunidad valenciana, y al oeste con Aragón. En el territorio catalán habitan en la actualidad 7.504.008 personas. Constituye un territorio muy denso y altamente industrializado. Su economía es la más importante de España, genera el $18,8 \%$ del producto interno bruto (PIB) español. 
buscó representación geográfica de Cataluña (de las cuatro provincias), escuela pública-concertada, diferentes niveles educativos, hombre/mujer, rural/urbano y diferentes edades.

La información obtenida brindó una visión suficiente, aunque siempre limitada, de las familias españolas y catalanas: su situación económica, de paro, riesgo de pobreza, abandono educativo, preocupaciones, inquietudes, dificultades, dudas... Los datos recogidos se ponderaron y equilibraron según las constantes y reiteraciones presentadas. La triangulación fue un elemento metodológico fundamental en la construcción del relato. Si bien gran parte de la información obtenida no aparece en los resultados - aunque es asimismo importante-, ha servido para construir el relato y ponderar toda la información.

\section{Datos cuantitativos y cualitativos}

Este apartado ofrece datos cuantitativos y cualitativos sobre la pobreza infantil y juvenil, la educación y, principalmente, el abandono escolar. La tabla $1^{9}$ permite tener una idea general del riesgo de pobreza o exclusión social en diferentes comunidades autónomas españolas.

En la tabla 1 se presenta el riesgo de pobreza o exclusión social y sus componentes por comunidades autónomas. La media en España, en 2009, era del $24,7 \%$, y en los siguientes años mostró un crecimiento débil y progresivo. El motivo del reducido incremento se debe a la fórmula para establecer el umbral de la pobreza. Durante los años de la crisis económica, las condiciones de vida empeoraron para una mayoría significativa de los ciudadanos españoles, debido a la reducción de los ingresos. También hay datos interesantes, como las grandes diferencias entre las comunidades autónomas. Destaca Ceuta, en 2014, con el 47,9 \% de la población en riesgo de pobreza. A continuación, Murcia y Andalucía, con el 44,9\% y el 42,3\%, respectivamente, en 2014. Cataluña tiene mejores datos (un 21,8 \% de la población), y Navarra y el País Vasco tienen la ratio más favorable (en 2014): 14,5 \% y 15,3\%, respectivamente. La tabla 2 hace referencia al riesgo de pobreza por tipo de hogar en todo el territorio español.

La tabla 2 muestra diferentes tipologías de familias españolas. En 2009, el 20,4 \% de la población estaba en riesgo de pobreza; en 2014, esta cifra ascendió al 22,2 \%. Los datos no son más elevados debido al progresivo descenso de los

9 La población en riesgo de pobreza o exclusión social es aquella que está en alguna de estas situaciones: en riesgo de pobreza (60\% mediana de los ingresos por unidad de consumo); en carencia material severa (con carencia en al menos cuatro conceptos de una lista de nueve); en hogares sin empleo o con baja intensidad de empleo (hogares en los que sus miembros en edad de trabajar lo hicieron menos del $20 \%$ del total de su potencia de trabajo durante el año de referencia). 
Tabla 1. Riesgo de pobreza o exclusión social por comunidades autónomas

\begin{tabular}{|l|c|c|c|c|c|c|}
\hline & $\mathbf{2 0 1 4}$ & $\mathbf{2 0 1 3}$ & $\mathbf{2 0 1 2}$ & $\mathbf{2 0 1 1}$ & $\mathbf{2 0 1 0}$ & $\mathbf{2 0 0 9}$ \\
\hline Nacional & 29,2 & 27,3 & 27,2 & 26,7 & 26,1 & 24,7 \\
\hline Andalucía & 42,3 & 38,3 & 36,9 & 38,4 & 35,0 & 33,3 \\
\hline Aragón & 20,7 & 19,8 & 18,4 & 18,6 & 17,5 & 12,6 \\
\hline Canarias & 37,0 & 35,5 & 40,3 & 37,8 & 35,7 & 38,2 \\
\hline Cataluña & 21,8 & 20,1 & 22,6 & 21,6 & 19,8 & 19,0 \\
\hline Ceuta & 47,9 & 47,0 & 38,4 & 33,6 & 35,9 & 37,3 \\
\hline Extremadura & 39,8 & 36,1 & 35,2 & 36,0 & 40,3 & 35,4 \\
\hline Extremadura & 19,2 & 20,1 & 19,8 & 18,6 & 19,3 & 19,6 \\
\hline
\end{tabular}

Fuente: elaboración propia con los datos de la Encuesta de Condiciones de Vida (ECD) del Instituto Nacional de Estadística (INE).

ingresos en las familias, haciendo que la media de la pobreza sea una cuantía económica menor. Esto hace que en dos años (2011-2013) disminuya el porcentaje de familias en riesgo de pobreza, aunque solo sea una cuestión estadística (hecho que se observa en los hogares de una sola persona). Corresponde en un número significativo a jubilados, los cuales muchos viven solos.

En 2009, a principios de la crisis, un 30,1 \% de estas personas estaban en riesgo de pobreza, pero curiosamente, con el avance de las dificultades económicas,

Tabla 2. Tasa de riesgo de pobreza por tipo de hogares

\begin{tabular}{|l|c|c|c|c|c|c|}
\hline & $\mathbf{2 0 1 4}$ & $\mathbf{2 0 1 3}$ & $\mathbf{2 0 1 2}$ & $\mathbf{2 0 1 1}$ & $\mathbf{2 0 1 0}$ & $\mathbf{2 0 0 9}$ \\
\hline Total & 22,2 & 20,4 & 20,8 & 20,6 & 20,7 & 20,4 \\
\hline $\begin{array}{l}\text { Hogares de una } \\
\text { persona }\end{array}$ & 20,7 & 19,2 & 20,6 & 27,9 & 28,9 & 30,1 \\
\hline $\begin{array}{l}\text { Dos adultos } \\
\text { sin niños } \\
\text { dependientes }\end{array}$ & 14,8 & 14,8 & 16,2 & 16,4 & 15,6 & 17,3 \\
\hline $\begin{array}{l}\text { Otros hogares } \\
\text { sin niños } \\
\text { dependientes }\end{array}$ & 15,6 & 11,8 & 12,2 & 13,1 & 10,7 & 7,9 \\
\hline $\begin{array}{l}\text { Un adulto con } \\
\text { uno o más niños } \\
\text { dependientes }\end{array}$ & 42,0 & 38,0 & 37,5 & 42,1 & 49,2 & 44,2 \\
\hline $\begin{array}{l}\text { Dos adultos con } \\
\text { uno o más niños } \\
\text { dependientes }\end{array}$ & 25,8 & 23,3 & 24,2 & 23,1 & 24,2 & 24,8 \\
\hline $\begin{array}{l}\text { Otros hogares } \\
\text { con niños } \\
\text { dependientes }\end{array}$ & 30,7 & 30,8 & 28,0 & 22,6 & 24,1 & 22,7 \\
\hline
\end{tabular}

Fuente: elaboración propia con los datos de la Encuesta de Condiciones de Vida (ECD) del Instituto Nacional de Estadística (INE). 
su situación mejoró, y en 2014 fue solo un 20,7 \%. El descenso ha sido muy destacado (en seis años fue casi del $10 \%$ ). Este colectivo no ha aumentado sus ingresos (en todo caso han disminuido), pero menos que otras tipologías familiares. Muchos de estos ciudadanos reciben la pensión de jubilación (en España, muy baja, pero segura hasta ahora), y una cantidad importante de trabajadores han visto reducidos sus ingresos de manera significativa o no tienen ingresos porque dejaron de trabajar. El umbral de pobreza es una cuantía más reducida, y los jubilados, al tener una paga baja, pero segura, ya no son un colectivo mayoritariamente pobre. En muchos casos, son el único ingreso fiable de toda la familia; todos sus miembros viven gracias a su paga.

Más datos interesantes hacen referencia a las tipologías familiares Dos adultos sin niños dependientes y Otros hogares sin niños dependientes; estas representan las organizaciones familiares con menos riesgo de pobreza, con mucha diferencia, en todos los años consultados. Por el contrario, las familias con hijos tienen más riesgo, pues en estas se destaca un adulto con uno o más niños dependientes: la tasa está por encima del $40 \%$ en todos los años, menos en 2012 y 2013. En 2009, un 44,2\% de este tipo de familias estaba en riesgo de pobreza; en 2010 (el peor año), la cifra ascendió al 49,2 \%, y 2014 descendió al 42,0 \%. Esta tipología de hogar tiene mayor riesgo de pobreza. La tabla 2 presenta un dato para la reflexión: aquellas familias con hijos tienen una mayor probabilidad de ser pobres. Por esta razón tiene sentido afirmar que la pobreza ha afectado fundamentalmente a los niños y a los jóvenes.

La tabla 3 muestra el riesgo de pobreza en menores de edad en 2014, con datos de Cataluña, España, la zona euro y la Unión Europea. Cataluña tiene la ratio más alta: una media del 38,4\%, con el 38,7\% en niños y el 37,5 \% en niñas. En España, el porcentaje es un poco más bajo: la media es del 35,1\%, con un 32,5 y el 38,1 \% de ellos y ellas, respectivamente. En la zona euro y en la Unión Europea los datos son parecidos: la media es del 26,5 y del $26,3 \%$, para uno y para otro. Puesto que los niños y los jóvenes están en una fase fundamental de su vida, en pleno proceso de crecimiento y aprendizaje, la propuesta es implementar políticas más profundas e intensas para paliar o moderar estas situaciones.

La educación está en cuestión en España y en todos los países; se considera que la institución no está a la altura de las circunstancias. Uno de los elementos más criticados es el abandono (y posterior fracaso) escolar de aquellos alumnos que no consiguen las credenciales mínimas y obligatorias. La tabla 4 presenta el abandono educativo temprano entre la población de 18 a 24 años (en diferentes países europeos) que no ha completado la educación secundaria obligatoria y no sigue ningún tipo de estudio o formación.

Las cifras difieren en función del país de referencia. La media de la Unión Europa (28 países) en 2003 era del 16,4 \% de los jóvenes; en 2008, del 14,7 \%, y en 2013, 
Tabla 3. Brecha del riesgo de pobreza en menores de 18 años en 2014

\begin{tabular}{|l|c|c|c|}
\hline & Hombres & Mujeres & Total \\
\hline Cataluña & 38,7 & 37,5 & 38,4 \\
\hline España & 32,5 & 38,1 & 35,1 \\
\hline Zona euro & 25,9 & 26,9 & 26,5 \\
\hline Unión Europea & 25,8 & 26,6 & 26,3 \\
\hline
\end{tabular}

Fuente: elaboración propia con los datos del Institut d'Estadística de Catalunya, a partir de la Encuesta de Condiciones de Vida (ECD) del Instituto Nacional de Estadística (INE). España, zona euro y Unión Europea: Eurostat.

Tabla 4. Abandono educativo temprano en Europa

\begin{tabular}{|l|c|c|c|}
\hline & $\mathbf{2 0 0 3}$ & $\mathbf{2 0 0 8}$ & $\mathbf{2 0 1 3}$ \\
\hline Unión Europea (28 países) & 16,4 & 14,7 & 12,0 \\
\hline Alemania & 12,8 & 11,8 & 9,9 \\
\hline Austria & 9,0 & 10,1 & 7,3 \\
\hline Bélgica & 14,3 & 12,0 & 11,0 \\
\hline Dinamarca & 10,4 & 12,5 & 8,0 \\
\hline Eslovenia & 4,6 & 5,1 & 3,9 \\
\hline España & 31,7 & 31,7 & 23,6 \\
\hline Finlandia & 10,1 & 9,8 & 9,3 \\
\hline Italia & 23,0 & 19,7 & 17,0 \\
\hline Malta & 49,9 & 27,2 & 20,8 \\
\hline
\end{tabular}

Fuente: elaboración propia a partir de las cifras de la educación en España. Curso 2012-2013 (ed. 2015).

del 12,0 \%. Las cifras muestran una reducción del porcentaje de jóvenes que abandonan los centros educativos temprano, sin la titulación obligatoria. Austria tiene muy buenas cifras: en 2003, solo el 9,0 \% abandonó la educación obligatoria; en 2008, el 10,1\%, y en 2013, el 7,3\%. Dinamarca también presenta unos índices por debajo de la media de la Unión Europea: en 2003 tenía el 10,4 \%; en 2008, el 12,5 \% y en 2013, el 8,0 \%. España, por el contrario, presenta unas cifras muy altas: en 2003, el 31,7 \%; en 2008, el 31,7 \%, y en 2013, el 23,6 \%. En dos años, el porcentaje fue el mismo, muy elevado y muy por encima de la media europea, pero cabe destacar la mejoría de los últimos años, porque el abandono se redujo significativamente: en cinco años, ocho puntos porcentuales.

Un país de referencia en educación como Finlandia presenta en 2003 el 10,1 \%; en 2008 , el 9,8 \%, y en 2013, el 9,3\%. Las cifras son estables, muy por debajo de 
la media europea, y mejoran de forma progresiva. Cabe destacar la capacidad de los malteses para transformar una tendencia negativa; el brusco descenso del abandono escolar se refleja en las siguientes cifras: en 2003 tenía el 49,9\%; en 2008 , el 27,2 \%, y en 2013, el 20,8 \%. La tabla 5 muestra el porcentaje de abandono educativo entre la población de 18 a 24 años que no ha completado el nivel de la educación secundaria obligatoria y no sigue ningún tipo de educación o formación en las comunidades autónomas españolas.

La media en España fue del 31,7 \% en 2003, y en 2013, del 23,6 \%, pero hay destacadas diferencias entre zonas. Resulta interesante destacar las Islas Baleares:

Tabla 5. Abandono educativo temprano en España

\begin{tabular}{|l|c|c|c|}
\hline & $\mathbf{2 0 0 3}$ & $\mathbf{2 0 0 8}$ & $\mathbf{2 0 1 3}$ \\
\hline Total & 31,7 & 31,7 & 23,6 \\
\hline Andalucía & 38,4 & 37,9 & 28,7 \\
\hline Aragón & 23,6 & 25,8 & 18,9 \\
\hline Balears (Illes) & 41,4 & 42,5 & 29,8 \\
\hline Cantabria & 28,9 & 22,9 & 12,1 \\
\hline Cataluña & 34,3 & 32,9 & 24,7 \\
\hline Galicia & 25,0 & 23,6 & 20,2 \\
\hline Madrid (Comunidad de) & 23,0 & 27,1 & 19,7 \\
\hline Murcia & 42,8 & 40,6 & 26,3 \\
\hline País Vasco & 15,9 & 14,8 & 9,9 \\
\hline Rioja (La) & 35,1 & 37,0 & 21,7 \\
\hline Ceuta / Melilla & 40,4 & 39,6 & 33,3 \\
\hline
\end{tabular}

Fuente: elaboración propia a partir de las cifras de la educación en España. Curso 2012-2013 (ed. 2015).

en 2003, fue del 41,4 \%; en, 2008, del 42,5\%, y en 2013, del 29,8 \%. La causa del alto abandono en las Islas Baleares se debe al impacto del turismo y la facilidad de encontrar trabajo y buenos salarios. Muchos jóvenes prefieren trabajar y dejar los estudios, ya que el mercado laboral los integra. Si bien el índice de abandono escolar se redujo a lo largo de los años, en plena crisis económica, en 2013, aún continuaba alto. La economía balear depende principalmente del turismo, y no ha habido un descenso significativo; todavía resulta fácil encontrar trabajo. En cambio, la situación en Andalucía es diferente. La tasa en 2003 era del 38,4 \%; en 2008 , del 37,9 \%, y en 2013, del 28,7 \%. Esta comunidad autónoma no tiene un sector empresarial potente. El sector inmobiliario, entre 2003 y 2008, era el principal motor de creación de empleo en la comunidad de Andalucía.

La crisis deja a muchos trabajadores en el paro, y, en consecuencia, muchos jóvenes solo pueden continuar estudiando, pues no tienen la opción de trabajar. 
Cataluña presenta la misma dinámica social: en los primeros años, el índice de abandono fue muy alto, debido al fácil acceso al mercado laboral; pero con la llegada de la crisis y el aumento del paro, los jóvenes continuaron en el sistema educativo, muchas veces en contra de su voluntad. En Cataluña, el índice de abandono educativo temprano en 2003 fue del 34,3 \%; en 2008, del 32,9\%, y en 2013, del $24,7 \%$.

Algunos de los problemas de la economía española son la baja calidad del empleo, los bajos sueldos y la escasa formación de los empleados. En periodos de bonanza económica, los jóvenes pueden encontrar fácilmente trabajo y abandonan los estudios. En cambio, en situaciones más complejas, cuando se requiere más creatividad e innovación, los trabajadores tienen muchas dificultades de empleabilidad, pues no tienen las capacidades adecuadas para comprender y situarse en un contexto turbulento.

La tabla 6 expone la tasa de abandono escolar según el quintil ${ }^{10}$ de renta disponible per cápita del hogar en 2010. La renta familiar es un elemento importante para determinar el itinerario académico de los hijos. Resulta difícil encontrar investigaciones que crucen estas variables: abandono escolar y renta familiar; para analizarlas, se requiere estadísticas alternativas, como la Encuesta de Condiciones de Vida (ECV):

Tabla 6. Tasa de abandono según el quintil de renta disponible per cápita del hogar en 2010 (incluyendo el alquiler imputado)

\begin{tabular}{|l|l|}
\hline Q1 & $\mathbf{3 5 , 0}$ \\
\hline Q2 & 32,3 \\
\hline Q3 & 23,4 \\
\hline Q4 & 17,5 \\
\hline Q5 & 10,2 \\
\hline
\end{tabular}

Fuente: elaboración propia con los datos de la Encuesta de Condiciones de Vida (ECV) del Instituto Nacional de Estadística (INE).

La tabla 6 ofrece datos muy valiosos sobre la relación entre capacidad económica familiar y abandono escolar temprano. El primer quintil, aquellas familias con la renta más baja, presenta el índice de abandono más alto, en el $35,0 \%$ de los casos. En el segundo se reduce al 32,3\%, hasta llegar al último, el quinto, aquellas familias con los ingresos más elevados, con el 10,2\% de abandono escolar temprano. Los datos son claros y evidentes, hay una relación directa entre renta y mayor o menor permanencia en los centros educativos.

10 Q1: renta per cápita hasta de 5309 euros; Q2: 5309-7485; Q3: 7485-9906; Q4: 9906-13.729; Q5: desde 13.72 en adelante. El alquiler imputado se aplica a los hogares que no pagan un alquiler completo por ser propietarios o por ocupar una vivienda alquilada a un precio inferior al del mercado o a título gratuito. El valor que se imputa es el equivalente al alquiler que se pagaría en el mercado por una vivienda similar a la ocupada, menor que cualquier alquiler realmente abonado. 
El abandono en España muestra un comportamiento claramente diferenciado según la capacidad económica de la familia. La tabla 7 analiza la capacidad de las familias españolas de llegar a fin de mes (es decir, tener un sueldo que les permita "llegar a fin de mes" sin restricciones materiales), y la relaciona según la tasa de abandono escolar.

Tabla 7. Tasa de abandono según la capacidad del hogar de llegar a fin de mes. 2010

\begin{tabular}{|l|c|}
\hline \multicolumn{1}{|c|}{ Con mucha dificultad } & $\mathbf{4 4 , 5}$ \\
\hline Con dificultad & 34,4 \\
\hline Con cierta dificultad & 21,4 \\
\hline Con cierta facilidad & 11,6 \\
\hline Con facilidad & 10,2 \\
\hline Con mucha facilidad & 7,0 \\
\hline
\end{tabular}

Fuente: elaboración propia con los datos de la Encuesta de Condiciones de Vida (ECV) del Instituto Nacional de Estadística (INE).

La tabla 7 presenta datos concluyentes al relacionar la tasa de abandono o permanencia en la escuela con las capacidades económicas de las familias españolas. Entre los hogares con muchas dificultades para llegar a fin de mes, un 44,5\% de sus hijos abandonan la escuela antes de tener las credenciales mínimas; entre aquellos que llegan con dificultad, un 34,4\% abandona la educación obligatoria, y entre aquellas que llegan con mucha facilidad, solo el 7,0 \% de sus hijos abandonan sus estudios antes de tener la titulación mínima obligatoria.

Las tablas 6 y 7 se complementan al situar la relación entre renta y abandono escolar. Asimismo, marcan un aspecto importante de la educación en España y una de las causas del fracaso escolar: la situación de pobreza y riesgo de exclusión social de las familias. Estas condiciones de escasez no solo tienen implicaciones en el aspecto material; también, y no menos importante, generan estados de malestar e inseguridad en la familia. Muchas veces las relaciones entre los adultos de la familia, y estos y sus hijos, se ven afectadas negativamente. Los padres se muestran más irascibles y reaccionan de forma más inconsistente e incontrolada (Marí-Klose y Marí-Klose, 2012).

Hasta el momento se han presentado datos cuantitativos sobre la pobreza de los hogares en España y el abandono educativo temprano. A continuación, se pasa de la visión general (macro) a la específica (micro), con aportaciones, siempre limitadas, que permiten conocer situaciones concretas reales, con descripciones individuales pero representativas de un amplio número de familias e hijos. Los siguientes son fragmentos de entrevistas en profundidad a padres y profesores. Todos los participantes son catalanes. 
Ramona, de 36 años, madre de Lleida, expone los problemas económicos de la familia y las dificultades para educar a su hija:

- Tengo una niña de 10 años y la educación de nuestros hijos es muy difícil. Todos trabajamos y no podemos estar el tiempo suficiente con ella. Yo ahora trabajo por las tardes y mi marido está parado. Cuando llego a casa, a las nueve de la noche, le tengo que revisar la agenda. La niña cada día está más rebelde, a mí no me hace caso. Entonces me pongo nerviosa y me empiezo a poner histérica. Hace unos años cogí una depresión.

- ¿Por qué?

- Porque la niña me superaba. Mi marido trabajaba llevando un camión y pasaba muchos días fuera de casa. Estaba hasta una semana fuera de casa. Entonces todo lo tenía que hacer yo, y, como no podía, cogí una depresión.

$-¿ Y$ ahora?

- Ahora hay otros problemas, está el paro. Tenemos dificultades económicas y todo se complica. La maestra me dijo el otro día que la niña no para en clase, que está muy nerviosa. Que tenía que ir al psicólogo. La verdad es que la situación muchas veces me desborda, ¡muchas veces! Nadie me ayuda... Ahora tiene 10 años, pero cuando tenga 12 o 13, ¿no sé lo que haré? ¡Alguien debería ayudar a los padres! (Comunicación personal, 11 de diciembre de 2010)

Marí-Klose (2012) afirma que la situación financiera de las familias es un elemento que afecta el desempeño de los niños en sus estudios, pero hay otros, psicológicos y sociales, que pueden crear estados de malestar e inseguridad que se trasladan a los hijos. La niña en clase está muy nerviosa, y la maestra le aconsejó a la madre llevarla al psicólogo. A esta, la situación la desborda, y comienza a pensar en su futuro, cuando la niña sea mayor; cuando llegue a la adolescencia, no podrá controlar la situación. Muchas familias viven en silencio circunstancias difíciles sin tener el apoyo social adecuado. Ramona considera que alguien debería ayudar a los padres.

Clara, madre de 30 años, de Barcelona, explica la situación de necesidad de algunas familias de su barrio (sabe de niños que no realizan alguna comida):

Yo vivo en un barrio de Barcelona donde hay mucha pobreza. Nosotros vamos tirando ${ }^{11}$, como podemos. Mi marido y yo trabajamos, de aquella manera, pero vamos tirando. Nos pagan muy poquito, pero no me 
quejo. Mucha gente está peor. Sé de familias en las que todos están en el paro, nadie trabaja. Cobran un poquito de ayuda del Estado, pero muy poquito. Creo que algunos no cobran nada. ¡La verdad que es una pena! Ves a los niños por la calle, con esa carita y todo esto. Sé que estas familias pasan necesidad y nadie los ayuda, ¡nadie! También en el barrio hay gente que no tiene luz, se la han cortado. ¡Imagínate! Pa' qué contarte más, me da mucha pena y nadie hace nada. (Comunicación personal, 23 de octubre de 2010)

Berta, maestra de inglés, de 40 años, ejerce en una escuela pública de Girona, y explica las situaciones de necesidad de sus alumnos:

- En la escuela cada vez más se ven las necesidades de casa. Les pedimos a los niños que sepan leer, escribir, hacer sumas, restas... pero nos encontramos que hay alumnos que no tienen las necesidades básicas cubiertas. Hace un mes, más o menos, le dije a la psicopedagoga que viene a la escuela que mirara a un niño que no acaba de aprender a leer ni a escribir. Te estoy diciendo que mi tutoría es de tercero de primaria. Cuando la psicopedagoga acabó la evaluación, vino a hablar conmigo. “¿Cómo ha ido?”, le pregunté, y me preguntó si el niño llevaba almuerzo a la escuela y si se quedaba en el comedor escolar. La verdad es que no le había dado importancia, pero [...] en muchos días los demás le daban parte de su almuerzo, o él iba a la cocina del centro a ver si había fruta. Me comentó que el niño solo le hablaba de las cosas que le gustaba comer e hizo un dibujo de él comiendo y cenando mucha comida. ¡Todos, él y sus padres! Me comentó que cuando los niños pasan necesidades básicas, difícilmente están atentos y predispuestos a aprender, ya que tienen otros problemas.

— ¿Habías hecho alguna tutoría antes con la familia?

- No. Ni la tutora del curso anterior había podido conseguir que vinieran nunca. El niño tiene una situación familiar complicada, padres separados. El niño a veces vive con el padre y con una nueva pareja, con la que tiene dos hijos; otras veces vive con la madre. Esta se dedica a trabajos de noche, y el niño se queda solo, muchas veces en casa o con alguna amiga de la madre. En fin... ¡imagínate qué situación... para que le importe aprender a leer y escribir con los problemas que tiene el pobre crío! (Comunicación personal, 11 de noviembre de 2010)

La maestra de inglés explica una realidad social poco conocida de los centros educativos: niños que no tienen las necesidades básicas cubiertas. La escuela no tiene los recursos adecuados para abordar estas situaciones, por lo cual necesita el apoyo de otras instituciones y un abordaje global. El equipo directivo de un instituto de Tarragona (un grupo de discusión) expone su mirada: 
Regina - Las dificultades en secundaria son cada vez mayores. Nos encontramos situaciones familiares dramáticas. Te podría poner muchos ejemplos, uno de tantos, uno de nuestros alumnos empezó a bajar las notas. Sabemos que en casa tenían necesidades, pero no sabíamos que estaban en una situación tan pésima. El tutor habló con el niño, también con su madre, para saber qué estaba pasando. Este alumno siempre iba aprobando, no con notas muy altas, pero sí con bienes y notables. La madre no me decía gran cosa en las tutorías, el alumno tampoco...

Joaquín - ¿Te acuerdas? Un día me llamó al instituto la trabajadora social del ayuntamiento, para preguntarme por este alumno en concreto, si habíamos notado alguna cosa, si la familia respondía... Me comentó que hacía un mes que les habían cortado el agua y la luz. Servicios sociales se habían involucrado en el caso. Los problemas familiares eran grandes.

- ¿Y qué pasó?

Marina - Estuvieron unas semanas más sin suministro, ahora me parece que están mejor. El chico continúa en el instituto. Aquel curso al final lo repitió, porque no se presentó a los exámenes en el segundo ni en el tercer trimestre. Ahora hace lo que puede. (Comunicación personal, 20 de septiembre de 2010)

Irene, profesora psicopedagoga en un instituto cerca de Barcelona, explica algunas vivencias del instituto:

Tenemos algunas familias en el instituto que durante algunas épocas del año desaparecen. Son familias con pocos recursos económicos y que se van moviendo de un sitio a otro, dependiendo de la uva, de la oliva... Hay dos hermanas que son buenas estudiantes, pero cuando marchan, marchan todos, todas las manos de la familia, sean pequeños o grandes. Estas niñas marchan con sus padres a trabajar desde los 6-7 años. Ellas ya lo tienen asumido, pero se dan cuenta de que si estuvieran siempre en el mismo sitio, podrían ir mejor en el instituto. Las dos han repetido, una en primaria y la otra en secundaria. Es una pena, porque son aplicadas y siempre tienen mucha predisposición en todo, pero si están uno o dos meses fuera, luego se sienten descolgadas. Nosotros ya les preparamos trabajos para hacer, pero no es lo mismo. (Comunicación personal, s. f.) 


\section{Conclusiones}

Este artículo ha presentado una realidad social conocida, pero poco explicitada. Algunos profesores ignoraban la situación vital de sus alumnos, pero, cuando la conocieron, comprendieron su comportamiento. Muchas familias españolas y catalanas viven en la pobreza, pero los menores, debido a su vulnerabilidad, sufren más estas situaciones. Elzo (2000) señala las dificultades de la institución familiar en la sociedad actual y destaca los cambios en la estructura familiar y la aparición de múltiples tipologías. Los datos cuantitativos muestran que aquellas familias con hijos tienen más dificultades y sufren más.

La educación es una disciplina muy estudiada, desde diferentes ámbitos, pero la relación entre pobreza y escuela ha sido escasamente tratada. Pocas investigaciones actuales han abordado este objeto de estudio de forma prioritaria, por lo que el análisis que aquí se presenta pretende llenar este vacío e indagar las posibles relaciones entre pobreza y educación.

En los últimos años, la institución educativa ha sido muy cuestionada; desde diferentes ámbitos, se le ha criticado por no estar a la altura de las circunstancias y por no realizar de manera correcta sus funciones. Innumerables veces se le ha culpado de los desajustes y problemas sociales. En España, y también en otros países, hay un número muy elevado de alumnos sin las credenciales mínimas obligatorias, considerados fracasados escolares. En 2013, un 23,6 \% de los jóvenes entre 18 y 24 años no había completado el nivel de educación secundaria obligatoria, jóvenes que, según Fernández Enguita (2010), tendrán muchas dificultades para integrarse en una sociedad avanzada y del conocimiento. Muchos estudios otorgan la responsabilidad a los profesores (de no tener la capacidad, la formación ni la motivación adecuadas); sitúan la reflexión solo en el marco educativo, como Hargreaves (1999), pero muchas circunstancias están más allá de la escuela y exigen una mirada global e integral. El abordaje de la educación exige la participación y la colaboración de todos los ciudadanos e instituciones.

Ante la crisis económica en España, las recetas del Gobierno para abordar los problemas monetarios consisten en aplicar políticas neoliberales con miras a limitar el estado del bienestar y, a su vez, los derechos de los ciudadanos. Giddens (2000) señala que el Estado ha perdido parte de su soberanía; en los momentos de máxima necesidad, no cumple con sus funciones, no acompaña a los ciudadanos y deja a muchas familias en la pobreza. Allepuz (2014) afirma que la pobreza infantil es una de las realidades más preocupantes de la situación social actual y del futuro.

Además de la literatura, se han presentado testimonios significativos que explican algunos estados de pobreza. Uno de estos es el de Clara, residente en Barcelona, quien explica las dificultades de familias y niños cuyas necesidades básicas no están cubiertas. Beck (1998) considera que en la sociedad actual amplios sectores viven en la incertidumbre, en condiciones precarias. 
Marí-Klose (2012) explica algunas consecuencias de la pobreza familiar que van más allá de la escasez material, y que tienen relación con el desarrollo psicológico de los adultos y los niños. Estos problemas generan malestar e inseguridad en los adultos y se traslada este estado a los hijos, por lo cual la relación tiende a ser más tensa y conflictiva. La pobreza infantil también tiene consecuencias en el desarrollo cognitivo y en los resultados educativos y facilita comportamientos antisociales.

Maslow (1991) sitúa en la satisfacción de las necesidades fisiológicas la base del desarrollo intelectual. Tener las necesidades básicas cubiertas es fundamental para un desarrollo cognitivo satisfactorio. Cuando hay necesidad alimentaria, todas las energías vitales buscan saciarla y los otros intereses pasan a un segundo plano.

Los datos que conectan la tasa de abandono escolar con la capacidad de las familias de llegar a final de mes muestran una relación directamente proporcional entre las dificultades económicas y el abandono escolar. En otras palabras, la pobreza de las familias es una de las causas relevantes de los bajos resultados educativos de muchos alumnos.

Estas circunstancias están más allá de los centros educativos y requieren un abordaje integral. Los ciudadanos, el Gobierno y todas las instituciones deberían implementar políticas públicas audaces y reales para fomentar la igualdad de oportunidades de todos los niños. No se debería tolerar la pobreza infantil y juvenil en ningún caso.

\section{Referencias}

Ander-Egg. E. (2000). Métodos y técnicas de investigación social. Buenos Aires: Lumen-Humanitas.

Allepuz, R. y Rosell, M. J. (2014). Anatomía de la pobreza a Catalunya. Lleida: Pagés.

Bauman, Z. (2003). Modernidad líquida. México, D. F.: Fondo de Cultura Económica.

Beck, U. (1998). La sociedad del riesgo: hacia una nueva modernidad. Barcelona: Paidós.

Castells, M. (2001). La galaxia internet. Barcelona: Plaza \& Janés.

Elzo, J. (2008). La voz de los adolescentes. Madrid: PPC.

Euroestat. (2014). Early leavers from education and training by sex. Recuperado de http://ec.europa.eu/eurostat/tgm/table.do?tab=table\&init=1\&language=en \&pcode=t2020_40\&plugin $=1$

Fernández Enguita, M. (2004). La escuela a examen. Madrid: Pirámide.

Fernández Enguita, M. (2010). Fracaso y abandono escolar en España. Barcelona: Fundación la Caixa. 
García de Blas, E. (11 de abril de 2014). España lidera el abandono escolar temprano en Europa con su mejor dato. El País. Recuperado el 11 de diciembre de 2015 de http://sociedad.elpais.com/sociedad/2014/04/11/ actualidad/1397211917_985641.html

Giddens, A. (2000). Un mundo desbocado: los efectos de la globalización en nuestras vidas. Madrid: Taurus.

Griggs, J. y Walker, R. (2008). The cost of child poverty for individuals and society. Nueva York: Joseph Rountree Foundation.

Hargreaves, A. (1999). Profesorado, cultura y postmodernidad. Madrid: Morata.

Halsey, A. H., Heath, A. y Ridge, J. (1980). Origins and destinations: family, class and education in modern Britain. Oxford: Oxford University Press.

Lipovetsky, G. (2008). La sociedad de la decepción. Barcelona: Anagrama.

Marí-Klose, P. y Marí-Klose, M. (2012). Crisi i pobresa infantil a Catalunya. Barcelona: Obra Social la Caixa.

Maslow, A. (1991). Motivación y personalidad. Madrid: Díaz de Santos.

Stake, R. E. (1998). Investigación con estudio de casos. Madrid: Morata.

Tahull, J. (2012). La modernitat i la postmodernitat. Anàlisi sociològica del concepte d'autoritat al sistema educatiu català (tesis inédita de doctorado) Universidad de Lleida, Lleida, España.

Tahull, J. y Montero, Y. (2013). Reflexionando sobre el concepto de autoridad. Revista de la Asociación de Sociología de la Educación, 6(3).

Tahull, J. y Montero, Y. (2013). Fundamentos de la posmodernidad. Limitaciones de la educación. Revista Arena 3(3).

Tahull, J. y Montero, Y. (2015). Malestar en la escuela. Conflicto entre profesores. Educar, 51(1), 169-188.

Tahull, J., Molina, F. y Montero, Y. (2016). Perspectives de l'autoritat del professorat a l'educació actual. Com veuen l'escola els seus protagonistes? Lleida: Universitat de Lleida.

Terrén, E. (1999). Educación y modernidad: entre la utopía y la burocracia. A Coruña: Anthropos. 